\title{
Clinical Data Analysis: A Challenging Career Option to Clinical Pharmacy Students
}

\section{Dear Readers,}

On behalf of the entire team of ijopp, I thank the authors for contributing their articles in this issue, and the reviewers involved in the publication process.

This is my first editorial in which I wish to discuss one of the most important areas of drug development process i.e. Data Analysis.

Pharmaceutical industry has been facing many challenges since years such as global competition, the need to speed up the drug development process and increasing expectations of FDA in the process and analytical development.

In clinical trials, case report form (CRF) is used to collect clinical trial data. The collected data are stored in a corresponding database which will be cleaned, processed, reported in the study report and archived using appropriate computerised tools. These tools support various aspects of the research process, such as trial design, data entry, source document tracking, data cleaning, analysis and reporting.

The integration, analysis and reporting of data collected in clinical trials is one of the critical paths in drug development. Challenges arrive while integrating data from multiple sources.

In the past, clinical trials have used only structured, clinically-sourced data, which was relatively easy to organize. Today, with data growing exponentially and getting more and more heterogeneous, pharma industries are facing significant challenges with data integration, data transformation, data cleansing and many other processes. But, with the advent of the new technologies, connected to devices for remote monitoring of trial patient participants, and advanced technologies it is even possible to analyse very large amounts of data with ease.

There are three main data analytics tools, namely SAS, R and Python that are most commonly opted.
SAS has been the undisputed leader in commercial analytics and offers a wide range of statistical tools and incredible technical support. It is not open source and hence the most expensive platform in the market, though it has the latest statistical functions to justify the price.

$\mathbf{R}$ is the open source alternative to SAS and mostly holds ground in academics and research. It capitalizes on its open source nature by making available the latest techniques very fast. It is a very cost effective option and there is extensive documentation available online for anyone seeking to master the platform.

Python is another open source scripting language that has grown to encompass libraries and functions for most statistical operations and model building.

Data analysts apply their specialized knowledge of data acquisition, management, analysis, and interpretation directly to healthcare data, providing actionable insights that physicians, clinical researchers, decisionmakers, and others can use. Many pharmacy professionals assume that learning new technology is not possible at this stage, even though they are capable and have analysis bent of mind. An interest in the potential for developing skills can manifest any time. It's a great career choice for those who want to apply healthcare expertise in a novel and innovative way.

I anticipate that you will find the information presented in this editorial to be intriguing, thought-provoking and useful in reaching new milestones in your career.

ijopp is currently accepting manuscripts for upcoming issues based on original qualitative or quantitative research. Please recommend the journal to your colleagues and students to make this endeavour meaningful.
DOI: 10.5530/ijopp.12.2.16

Address for correspondence: Dr. Ramjan Shaik, Editor, ijopp. Email Id: ramjanshaik@gmail.com 